\title{
On the Derivative of a Polynomial
}

\author{
Nisar A. Rather, Mushtaq A. Shah \\ P.G. Department of Mathematics, Kashmir University, Srinagar, India \\ Email: \{dr.narather, mushtaqa022\}@gmail.com
}

Received May 2, 2012; revised June 2, 2012; accepted June 9, 2012

\begin{abstract}
Certain refinements and generalizations of some well known inequalities concerning the polynomials and their derivatives are obtained.
\end{abstract}

Keywords: Polynomials; Inequalities; Complex Domain

\section{Introduction to the Statement of Results}

Let $P_{n}(z)$ denote the space of all complex polynomials

$$
\begin{array}{r}
P(z)=\sum_{j=1}^{n} a_{j} z^{j} \text { of degree } n \text {. If } P \in P_{n} \text {, then } \\
\max _{|z|=1}\left|P^{\prime}(z)\right| \leq n \max _{|z|=1}|P(z)|
\end{array}
$$

and

$$
\max _{|z|=R>1}|P(z)| \leq R^{n} \max _{|z|=1}|P(z)| .
$$

Inequality (1) is an immediate consequence of S.Bernstein's theorem (see [1]) on the derivative of a trigonometric polynomial. Inequality (2) is a simple deduction from the maximum modulus principle (see [2, p. 346] or $[3$, p. 137]).

Both the inequalities (1) and (2) are sharp and the equality in (1) and (2) holds if and only if $P(z)$ has all its zeros at the origin. It was shown by Frappier, Rahman and Ruscheweyh [4, Theorem 8] that if $P \in P_{n}$, then

$$
\max _{|z|=1}\left|P^{\prime}(z)\right| \leq n \max _{1 \leq k \leq 2 n}\left|P\left(e^{i k \pi / n}\right)\right| \text {. }
$$

Clearly (3) represents a refinement of (1), since the maximum of $|P(z)|$ on $|z|=1$ may be larger than the maximum of $|P(z)|$ taken over $(2 n)^{\text {th }}$ roots of unity, as is shown by the simple example $P(z)=z^{n}+i a$, $a>0$.

A. Aziz [5] showed that the bound in (3) can be considerably improved. In fact proved that if $P \in P_{n}$, then for every given real $\alpha$,

$$
\max _{|z|=1}\left|P^{\prime}(z)\right| \leq \frac{n}{2}\left(M_{\alpha}+M_{\alpha+\pi}\right)
$$

where

$$
M_{\alpha}=\max _{1 \leq k \leq n}\left|P\left(e^{i(\alpha+2 k \pi) / n}\right)\right|
$$

and $M_{\alpha+\pi}$ is obtained by replacing $\alpha$ by $\alpha+\pi$. The result is best possible and equality in (4) holds for $P(z)=z^{n}+r e^{i \alpha},-1 \leq r \leq 1$.

Clearly inequality (4) is an interesting refinement of inequality (3) and hence of Bernstein inequality (1) as well.

If we restrict ourselves to the class of polynomials $P \in P_{n}$ having no zero in $|z|<1$, then the inequality (1) can be sharpened. In fact, P. Erdös conjectured and later P. D. Lax [6] (see also [7]) verified that if $P(z) \neq 0$ for $|z|<1$, then (1) can be replaced by

$$
\max _{|z|=1}\left|P^{\prime}(z)\right| \leq \frac{n}{2} \max _{|z|=1}|P(z)| .
$$

In this connection A. Aziz [5], improved the inequality (4) by showing that if $P \in P_{n}$ and $P(z)$ does not vanish in $|z|<1$, then for every real $\alpha$,

$$
\max _{|z|=1}\left|P^{\prime}(z)\right| \leq \frac{n}{2}\left(M_{\alpha}^{2}+M_{\alpha+\pi}^{2}\right)^{1 / 2}
$$

where $M_{\alpha}$ is defined by (5). The result is best possible and equality in (7) holds for $P(z)=z^{n}+e^{i \alpha}$.

A. Aziz [5] also proved that if $P \in P_{n}$ and $P(z) \neq 0$ in $|z|<1$, then for every real $\alpha$ and $R>1$,

$$
\max _{|z|=1}|P(R z)-P(z)| \leq \frac{R^{n}-1}{2}\left(M_{\alpha}^{2}+M_{\alpha+\pi}^{2}\right)^{1 / 2}
$$

In this paper, we first present the following result which is a refinement of inequality (7).

Theorem 1. If $P \in P_{n}, P(z)$ does not vanish in $|z|<1$ and $m=\min _{|z|=1}|P(z)|$, then for every real $\alpha$,

$$
\max _{|z|=1}\left|P^{\prime}(z)\right| \leq \frac{n}{2}\left(M_{\alpha}^{2}+M_{\alpha+\pi}^{2}-2 m^{2}\right)^{1 / 2} .
$$

where $M_{\alpha}$ is defined by (5). The result is best possible and equality in (9) holds for $P(z)=z^{n}+e^{i \alpha}$. 
As an application of Theorem 1, we mention the corresponding improvement of (8).

Theorem 2. If $P \in P_{n}$, and $P(z) \neq 0$ for $|z|<1$ and $m=\min _{|z|=1}|P(z)|$ then for every real $\alpha$ and $R>1$,

$$
|P(R z)-P(z)| \leq \frac{R^{n}-1}{2}\left(M_{\alpha}^{2}+M_{\alpha+\pi}^{2}-2 m^{2}\right)^{1 / 2}
$$

where $M_{\alpha}$ is defined by (5). The result is best possible and equality in (10) holds for $P(z)=z^{n}+e^{i \alpha}$.

Here we also consider the class of polynomials $P \in P_{n}$ having no zero in $|z|<k, k>0$ and present some generalizations of the inequalities (9) and (10). First we consider the case $k \geq 1$ and prove the following result which is a generalization of inequality (9).

Theorem 3. If $P \in P_{n}$ does not vanish in $|z|<k$, $k \geq 1$ and $m=\min _{|z|=k}|P(z)|$, then for every real $\alpha$,

$$
\max _{|z|=1}\left|P^{\prime}(z)\right| \leq \frac{n}{\sqrt{2\left(1+k^{2}\right)}}\left(M_{\alpha}^{2}+M_{\alpha+\pi}^{2}-2 m^{2}\right)^{1 / 2}
$$

where $M_{\alpha}$ is defined by (5).

Next result is a corresponding generalization of the inequality (10).

Theorem 4. If $P \in P_{n}$ does not vanish in $|z|<k$, $k \geq 1$ and $m=\min _{|z|=k}|P(z)|$, then for every real $\alpha$ and $R>1$,

$$
|P(R z)-P(z)| \leq \frac{R^{n}-1}{\sqrt{2\left(1+k^{2}\right)}}\left(M_{\alpha}^{2}+M_{\alpha+\pi}^{2}-2 m^{2}\right)^{1 / 2}
$$

where $M_{\alpha}$ is defined by (5).

Remark 1. For $k=1$, Theorem 3 and Theorem 4 reduces to the Theorem 1 and Theorem 2 respectively.

For the case $k \leq 1$, we have been able to prove:

Theorem 5. If $P \in P_{n}, P(z)$ has no zero in $|z|<k$, $k \leq 1$ and $m=\min _{|z|=k}|P(z)|$, then for every real $\alpha$,

$$
\begin{aligned}
& \max _{|z|=1}\left|P^{\prime}(z)\right| \\
& \leq \frac{n}{\sqrt{2\left(1+k^{2 n}\right)}}\left(M_{\alpha}^{2}+M_{\alpha+\pi}^{2}-2 m^{2}\right)^{1 / 2}
\end{aligned}
$$

provided $\left|P^{\prime}(z)\right|$ and $\left|Q^{\prime}(z)\right|$ attain maximum at the same point on $|z|=1$ where $Q(z)=z^{n} \overline{P(1 / \bar{z})}$. The result is best possible and equality in (13) holds for $P(z)=z^{n}+k^{n}$.

Theorem 6. If $P \in P_{n}, P(z)$ has no zero in $|z|<k$, $k \leq 1$ and $m=\min _{|z|=k}|P(z)|$, then for every real $\alpha$ and $R>1$,

$$
|P(R z)-P(z)| \leq \frac{R^{n}-1}{\sqrt{2\left(1+k^{2 n}\right)}}\left(M_{\alpha}^{2}+M_{\alpha+\pi}^{2}-2 m^{2}\right)^{1 / 2},
$$

provided $\left|P^{\prime}(z)\right|$ and $\left|Q^{\prime}(z)\right|$ attain maximum at the same point on $|z|=1$ where $Q(z)=z^{n} \overline{P(1 / \bar{z})}$. The result is best possible and equality in (14) holds for $P(z)=z^{n}+k^{n}$.

\section{Lemmas}

For the proofs of these theorems, we need the following lemmas. The first Lemma is due to A. Aziz [5].

Lemma 1. If $P \in P_{n}$, then for $|z|=1$ and for every real $\alpha$,

$$
\left|P^{\prime}(z)\right|^{2}+\left|n P(z)-z P^{\prime}(z)\right|^{2} \leq \frac{n^{2}}{2}\left(M_{\alpha}^{2}+M_{\alpha+\pi}^{2}\right)
$$

where $M_{\alpha}$ is defined by (5).

Lemma 2. If $P \in P_{n}$ and $P(z) \neq 0$ for $|z|<k$, $k \geq 1$, then for $|z|=1$,

$$
k\left|P^{\prime}(z)\right| \leq\left|n P(z)-z P^{\prime}(z)\right|-n m
$$

where $m=\min _{|z|=k}|P(z)|$.

Lemma 2 is a special cases of a result due to A. Aziz and N. A. Rather [8, Lemma 5].

Lemma 3. If $P \in P_{n}$ does not vanish in $|z|<k$, $k \leq 1$, then

$$
k^{n}\left|P^{\prime}(z)\right| \leq \max _{|z|=1}\left|Q^{\prime}(z)\right| \text { for }|z|=1
$$

where $Q(z)=z^{n} \overline{P(1 / \bar{z})}$.

This Lemma is due to N. K. Govil [9].

Lemma 4. If $P(z)$ is a polynomial of degree $n$ which does not vanish in $|z|<k, \quad k \leq 1$, then for $|z|=1$

$$
k^{n}\left|P^{\prime}(z)\right|+n \min _{|z|=k}|P(z)| \leq \max _{|z|=1}\left|Q^{\prime}(z)\right|
$$

where $Q(z)=z^{n} \overline{P(1 / \bar{z})}$.

Proof of Lemma 4. Let $m=\min _{|z|=k}|P(z)|$. If $P(z)$ has a zero on $|z|=k$, then $m=0$ and the result follows from Lemma 3. Henceforth we assume that $P(z)$ has no zero on $|z|=k$, therefore $m>0$ and

$$
m \leq|P(z)| \text { for }|z|=k \text {. }
$$

If $\alpha$ is any real or complex number with $|\alpha|<1$, then for $|z|=k$,

$$
\left|\alpha m z^{n} / k^{n}\right|<|P(z)| .
$$

By Rouche's Theorem, it follows that the polynomial $F(z)=P(z)-\alpha m z^{n} / k^{n}$ does not vanish in $|z|<k$, for every real or complex number $\alpha$ with $|\alpha|<1$. Apply- 
ing Lemma 3 to the polynomial $F(z)$, we get

$$
k^{n}\left|F^{\prime}(z)\right| \leq \max _{|z|=1}\left|G^{\prime}(z)\right| \text { for }|z|=1 .
$$

where

$$
\begin{aligned}
G(z) & =z^{n} \overline{P(1 / \bar{z})}=z^{n} \overline{P(1 / \bar{z})}-\bar{\alpha} m / k^{n} \\
& =Q(z)-\bar{\alpha} m / k^{n} .
\end{aligned}
$$

Replacing $F(z)$ by $P(z)-\alpha m z^{n} / k^{n}$ and $G(z)$ by $Q(z)-\bar{\alpha} m / k^{n}$, we obtain from (16) for $|z|=1$,

$$
k^{n}\left|P^{\prime}(z)-n \alpha m z^{n-1} / k^{n}\right| \leq \max _{|z|=1}\left|Q^{\prime}(z)\right| .
$$

Now choosing the argument of $\alpha$ in the left hand side of (17) such that

$$
\left|P^{\prime}(z)-n \alpha m z^{n-1} / k^{n}\right|=\left|P^{\prime}(z)\right|+\left|n m \alpha / k^{n}\right|
$$

we obtain for $|z|=1$,

$$
k^{n}\left|P^{\prime}(z)\right|+|\alpha| n m \leq \max _{|z|=1}\left|Q^{\prime}(z)\right| .
$$

Letting $|\alpha| \rightarrow 1$, we get the desired result. This proves Lemma 4.

\section{Proof of the Theorems}

Proof of Theorem 1. By hypothesis $P(z)$ does not vanish in $|z|<1$ and $m=\min _{|z|=k}|P(z)|$, therefore, by Lemma 2 with $k=1$, we have

$$
\left(\left|P^{\prime}(z)\right|+n m\right)^{2} \leq\left|n P(z)-z P^{\prime}(z)\right|^{2} \text { for }|z|=1 .
$$

This gives with the help of Lemma 1

$$
\begin{aligned}
\left|P^{\prime}(z)\right|^{2}+\left(\left|P^{\prime}(z)\right|+n m\right)^{2} & \leq\left|P^{\prime}(z)\right|^{2}+\left|n P(z)-z P^{\prime}(z)\right|^{2} \\
& \leq \frac{n^{2}}{2}\left(M_{\alpha}^{2}+M_{\alpha+\pi}^{2}\right) .
\end{aligned}
$$

Since

$$
\begin{aligned}
\left(\left|P^{\prime}(z)\right|+n m\right)^{2} & =\left|P^{\prime}(z)\right|^{2}+n^{2} m^{2}+2 n m\left|P^{\prime}(z)\right| \\
& \geq\left|P^{\prime}(z)\right|^{2}+n^{2} m^{2},
\end{aligned}
$$

it follows that

$$
2\left|P^{\prime}(z)\right|^{2}+n^{2} m^{2} \leq \frac{n^{2}}{2}\left(M_{\alpha}^{2}+M_{\alpha+\pi}^{2}\right),
$$

which implies for $|z|=1$

$$
\left|P^{\prime}(z)\right| \leq \frac{n}{2}\left(M_{\alpha}^{2}+M_{\alpha+\pi}^{2}-2 m^{2}\right)^{1 / 2}
$$

and hence

$$
\max _{|z|=1}\left|P^{\prime}(z)\right| \leq \frac{n}{2}\left(M_{\alpha}^{2}+M_{\alpha+\pi}^{2}-2 m^{2}\right)^{1 / 2} .
$$

This completes the proof of Theorem 1 .
Proof of Theorem 2. Applying (2) to the polynomial $P^{\prime}(z)$ which is of degree $n-1$ and using Theorem 1 , we obtain for $t \geq 1$ and $0 \leq \theta<2 \pi$,

$$
\begin{aligned}
\left|P^{\prime}\left(t e^{i \theta}\right)\right| & \leq t^{n-1} \max _{|z|=1}\left|P^{\prime}(z)\right| \\
& \leq \frac{n}{2} t^{n-1}\left(M_{\alpha}^{2}+M_{\alpha+\pi}^{2}-2 m^{2}\right)^{1 / 2} .
\end{aligned}
$$

Hence for each $\theta, 0 \leq \theta<2 \pi$ and $R>1$, we have

$$
\begin{aligned}
\left|P\left(\operatorname{Re}^{i \theta}\right)-P\left(e^{i \theta}\right)\right| & =\left|\int_{1}^{R} e^{i \theta} P^{\prime}\left(t e^{i \theta}\right) \mathrm{d} t\right| \leq \int_{1}^{R}\left|P^{\prime}\left(t e^{i \theta}\right)\right| \mathrm{d} t \\
& \leq \frac{1}{2}\left(M_{\alpha}^{2}+M_{\alpha+\pi}^{2}-2 m^{2}\right)^{1 / 2} \int_{1}^{R} n t^{n-1} \mathrm{~d} t . \\
& =\frac{1}{2}\left(M_{\alpha}^{2}+M_{\alpha+\pi}^{2}-2 m^{2}\right)^{1 / 2}\left(R^{n}-1\right) .
\end{aligned}
$$

This implies for $|z|=1$ and $R>1$,

$$
|P(R z)-P(z)| \leq \frac{R^{n}-1}{2}\left(M_{\alpha}^{2}+M_{\alpha+\pi}^{2}-2 m^{2}\right)^{1 / 2},
$$

which proves Theorem 2 .

The proof of the Theorem 3 and 4 follows on the same lines as that of Theorems 1 and 2, so we omit the details.

Proof of Theorem 5. Since all the zeros of $P(z)$ lie in $|z| \geq k$, where $k \leq 1, \quad m=\min _{|z|=k}|P(z)|$, by Lemma 4 , we have

$$
k^{n} \max _{|z|=1}\left|P^{\prime}(z)\right|+n m \leq \max _{|z|=1}\left|Q^{\prime}(z)\right|,
$$

where $Q(z)=z^{n} \overline{P(1 / \bar{z})}$. Also by hypothesis $\left|P^{\prime}(z)\right|$ and $\left|Q^{\prime}(z)\right|$ become maximum at the same point on $|z|=1$, if

$$
\max _{|z|=1}\left|P^{\prime}(z)\right|=\left|P^{\prime}\left(e^{i \alpha}\right)\right|, 0 \leq \alpha<2 \pi,
$$

then

$$
\max _{|z|=1}\left|Q^{\prime}(z)\right|=\left|Q^{\prime}\left(e^{i \alpha}\right)\right|, 0 \leq \alpha<2 \pi
$$

and it can be easily verified that

$$
\left|Q^{\prime}(z)\right|=\left|n P(z)-z P^{\prime}(z)\right| \text { for }|z|=1 .
$$

Therefore, by Lemma 1

$$
\begin{aligned}
& \left|P^{\prime}\left(e^{i \alpha}\right)\right|^{2}+\left|Q^{\prime}\left(e^{i \alpha}\right)\right|^{2} \\
& =\left|P^{\prime}\left(e^{i \alpha}\right)\right|^{2}+\left|n P\left(e^{i \alpha}\right)-e^{i \alpha} P^{\prime}\left(e^{i \alpha}\right)\right|^{2} \\
& \leq \frac{n^{2}}{2}\left(M_{\alpha}^{2}+M_{\alpha+\pi}^{2}\right) .
\end{aligned}
$$

This gives with the help of (18), (19) and (20) that

$$
\begin{aligned}
& \left|P^{\prime}\left(e^{i \alpha}\right)\right|^{2}+\left(k^{n}\left|P^{\prime}\left(e^{i \alpha}\right)\right|+n m\right)^{2} \\
& \leq\left|P^{\prime}\left(e^{i \alpha}\right)\right|^{2}+\left|Q^{\prime}\left(e^{i \alpha}\right)\right|^{2} \leq \frac{n^{2}}{2}\left(M_{\alpha}^{2}+M_{\alpha+\pi}^{2}\right),
\end{aligned}
$$


which implies,

$$
\left|P^{\prime}\left(e^{i \alpha}\right)\right|^{2}+k^{2 n}\left|P^{\prime}\left(e^{i \alpha}\right)\right|^{2}+n^{2} m^{2} \leq \frac{n^{2}}{2}\left(M_{\alpha}^{2}+M_{\alpha+\pi}^{2}\right) .
$$

Equivalently,

$$
\left|P^{\prime}\left(e^{i \alpha}\right)\right|^{2} \leq \frac{n^{2}}{2\left(1+k^{2 n}\right)}\left(M_{\alpha}^{2}+M_{\alpha+\pi}^{2}-2 m^{2}\right)
$$

and hence

$$
\max _{|z|=1}\left|P^{\prime}(z)\right| \leq \frac{n}{\sqrt{2\left(1+k^{2 n}\right)}}\left(M_{\alpha}^{2}+M_{\alpha+\pi}^{2}-2 m^{2}\right)^{1 / 2} .
$$

This completes the proof of Theorem 5 .

Theorem 6 follows on the same lines as that of Theorem 2, so we omit the details.

\section{REFERENCES}

[1] A. C. Schaffer, "Inequalities of A. Markoff and S. Bernstein for Polynomials and Related Functions," Bulletin of the American Mathematical Society, Vol. 47, 1941, pp. 565-579. doi:10.1090/S0002-9904-1941-07510-5

[2] M. Riesz, "Uber Einen Satz des Herrn Serge Bernstein," Acta Mathematica, Vol. 40, 1916, pp. 337-347.
doi:10.1007/BF02418550

[3] G. Pólya and G. Szegö, “Aufgaben und lehrsätze aus der Analysis,” Springer-Verlag, Berlin, 1925.

[4] C. Frappier, Q. I. Rahman and St. Ruscheweyh, "New Inequalities for Polynomials," Transactions of the American Mathematical Society, Vol. 288, 1985, pp. 69-99. doi:10.1090/S0002-9947-1985-0773048-1

[5] A. Aziz, "A Refinement of an Inequality of S.Bernstein," Journal of Mathematical Analysis and Applications, Vol. 142, No. 1, 1989, pp. 226-235. doi:10.1016/0022-247X(89)90370-3

[6] P. D. Lax, "Proof of a Conjecture of P.Erdös on the Derivative of a Polynomial," Bulletin of the American Mathematical Society, Vol. 50, 1944, pp. 509-513. doi:10.1090/S0002-9904-1944-08177-9

[7] A. Aziz and Q. G. Mohammad, "Simple Proof of a Theorem of Erdos and Lax," Proceedings of the American Mathematical Society, Vol. 80, 1980, pp. 119-122.

[8] A. Aziz and N. A. Rather, "New $L^{q}$ Inequalities for Polynomials," Mathematical Inequalities and Applications, Vol. 2, 1998, pp. 177-191. doi:10.7153/mia-01-16

[9] N. K. Govil and Q. I. Rahman, "Functions of Exponential Type Not Vanishing in a Half Plane and Related Polynomials," Transactions of the American Mathematical Society, Vol. 137, 1969, pp. 501-517. doi:10.1090/S0002-9947-1969-0236385-6 